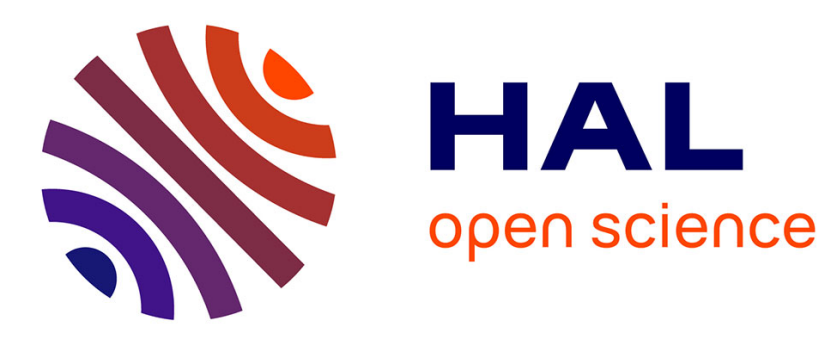

\title{
How Disorder Impacts Routing in Human-Centric Disruption Tolerant Networks
}

\author{
Anh-Dung Nguyen, Patrick Sénac, Michel Diaz
}

\section{To cite this version:}

Anh-Dung Nguyen, Patrick Sénac, Michel Diaz. How Disorder Impacts Routing in Human-Centric Disruption Tolerant Networks. The ACM SIGCOMM 2013 Workshop on Future Human-Centric Multimedia Networking (FhMN 2013), Aug 2013, Hong Kong SAR China. pp.47-52. hal-00908488

\section{HAL Id: hal-00908488 \\ https://hal.science/hal-00908488}

Submitted on 23 Nov 2013

HAL is a multi-disciplinary open access archive for the deposit and dissemination of scientific research documents, whether they are published or not. The documents may come from teaching and research institutions in France or abroad, or from public or private research centers.
L'archive ouverte pluridisciplinaire HAL, est destinée au dépôt et à la diffusion de documents scientifiques de niveau recherche, publiés ou non, émanant des établissements d'enseignement et de recherche français ou étrangers, des laboratoires publics ou privés. 


\section{How Disorder Impacts Routing in Human-Centric Disruption Tolerant Networks}

\author{
Anh-Dung Nguyen \\ ISAE-University of Toulouse \\ LAAS-CNRS \\ Toulouse, France \\ anh-dung.nguyen@isae.fr
}

\author{
Patrick Senac \\ ISAE-University of Toulouse \\ LAAS-CNRS \\ Toulouse, France \\ patrick.senac@isae.fr
}

\author{
Michel Diaz \\ LAAS-CNRS \\ Toulouse, France \\ michel.diaz@laas.fr
}

\begin{abstract}
Human-Centric DTNs exhibit some degree of regularity on their temporal contact patterns [1]. The impact of this regularity on network performances has not been well studied and analyzed. In this paper, we study this temporal dimension of Human-Centric DTNs and its impacts on routing performances. We propose a simple parametric network model which covers the full spectrum of contact patterns from strictly periodic to fully random ones. Based on this model, we study the impact of contact patterns regularity on routing performances and we show how to exploit the temporal structure to navigate with a good resource/performance tradeoff in Human-Centric DTNs. Simulation and analytical analysis show that efficient routing with respect to their degree of regularity emerge within a subset of dynamic networks. Moreover, we show there is a specific degree of regularity where routing performance achieves its optimum.
\end{abstract}

\section{Categories and Subject Descriptors}

C.2.1 [Network Architecture and Design]: Wireless communication; C.2.2 [Network Protocols]: Routing protocols

\section{Keywords}

Human-Centric DTNs, routing, search and retrieval, disorder, dynamic network structure

\section{INTRODUCTION}

The advent of new generations of wireless communication devices, coupled with the ever-increasing growth of multimedia contents, make multimedia services becoming one of the most resources-consuming use of the Internet. In this paper, we envision a network at the edge of the Internet in which human portable devices, endowed with peer-to-peer communication and sensing capacities, communicate each other without infrastructure to offer potentially free infrastruc-

Permission to make digital or hard copies of all or part of this work for personal or classroom use is granted without fee provided that copies are not made or distributed for profit or commercial advantage and that copies bear this notice and the full citation on the first page. Copyrights for components of this work owned by others than ACM must be honored. Abstracting with credit is permitted. To copy otherwise, or republish, to post on servers or to redistribute to lists, requires prior specific permission and/or a fee. Request permissions from permissions@acm.org.

Copyright 20XX ACM X-XXXXX-XX-X/XX/XX ...\$15.00. tureless new multimedia services. Because of their mobility nature, these networks might suffer from nodes and links churns, and hence can be classified within the scope of Delay/Disruption Tolerant Networks (DTNs). These HumanCentric DTNs, if successfully deployed, will play the role of a complement to infrastructure-based networks by freeing users from infrastructure dependence and reducing the load on the network core. These networks will pave the way for an pervasive computing environment to which users can access anywhere anytime. One can imagine many future applications of this type of network, e.g., content sharing and retrieval, gaming, recovery networks, offloading, etc.

The modeling and study of the properties of these dynamic networks under the umbrella of the science of dynamic and stochastic graphs is a recent scientific field when compared to the field of static networks and their underlying graph models [14]. Therefore there remains numerous open questions. One of the most interesting fundamental problem is the issue of efficient routing in such networks. Indeed, unlike routing in static networks with the support of the well developed static graph theory, there is still no widely developed theoretical background to understand deeply the routing issues in dynamic networks. In the context of DTNs, several routing algorithms and heuristics have been proposed [2], trying to answer some specific questions about routing while usually ignoring and not leveraging on the profound structural properties of dynamic networks.

In this paper, we aim to contribute to understanding the impact of the dynamic structure of Human-Centric DTNs on information routing. In DTNs, communication relies on intermittent contact between nodes and their mobility. Routing in such a network means finding a temporal path between a source node and a destination node [15]. Finding an efficient temporal path is made difficult by the lack of a-priori knowledge of the evolution of the dynamic network topology. In other words, nodes can base their forwarding decisions on their local knowledge based on a limited temporal and spatial scope only. In the context of acquaintance networks [20], it was shown that people implicitly solve the information routing problem by leveraging on spatial or structural properties of the network, such as nodes relative or absolute positions [7], nodes centrality [21], to enforce the forwarding decision. This approach can be extended to dynamic networks by inferring the spatio-temporal nodes relationships from their peer to peer interactions [18]. In [17], we have sketched out a new model to capture the disorder degree of dynamic networks and highlighted its impacts on routing in 
such networks. This paper investigates further this direction by giving the full details of the study in the first part and showing how one can exploit this disorder to make efficient routing in Human-Centric DTNs in the second part.

\section{RELATED WORKS}

With the potential of future pervasive mobile networks, recent researches have drawn attention to the theories of dynamic networks (see [4] a for a good review of recent researches on temporal networks). The persistence and regularity of communication patterns is one interesting aspect of dynamic networks. In [1], Clauset et al. investigate real dynamic network traces divided in time snapshots to demonstrate that dynamic networks have a high persistence and periodicity in time. They show that the daily and weekly rhythms of human social behaviors make periodic contacts patterns in the network. Motifs is another aspect of dynamic network which has drawn attention of researchers. In [9], Kovanen et al. formalize the notion of temporal motif as isomorphic connected subgraphs and propose an algorithm to find such motifs in a temporal graph. They apply this algorithm on mobile phone traces to study the behavior of mobile users.

In the context of human acquaintance networks, the famous experiment of Milgram [20] consists in sending a package from a group of persons living in Boston to a randomly chosen person in Massachusetts only through their acquaintances. The obtained result was striking in the fact that the chains of persons leading to the destination are very short, having on average only 6 peoples. This can be considered a typical example of routing in a social network. The routing problem we are addressing here is different. We consider Human-Centric DTNs in which nodes (i.e., mobile devices) can communicate only through peer-to-peer contacts (i.e., when they are in the radio range of each other) and there is no long range contact unlike the postal service in the Milgram case. Besides, nodes in the network may not know each other in advance.

Kleinberg [7] is interested in the algorithmic component of the experiment of Milgram, i.e. how individuals can find such short routes in such large social network. The author proposes a simple model that embeds the geographic world in a $2 \mathrm{D}$ lattice (the result can be generalized to other number of dimensions). Nodes have local contacts with neighboring nodes and choose a long-range contact with a far node with a probability inversely proportional to the distance to the later. The probability distribution follows a power law $d^{-\alpha}$ where $d$ is the distance and $\alpha$ is the unique parameter of the model. By applying a greedy algorithm in which nodes select the contact closest to the destination as message relay, the author demonstrates that the routing delay reaches its optimum (i.e., in logarithmic time) when $\alpha$ equals to 2 and is polynomial otherwise. This is due to the isotropic structure of the network at the optimum which establishes gradient allowing nodes to have a cue to find short paths. In [8], Kleinberg discusses various decentralized search algorithms.

Watts et al. [21] proposed another model for searching in social networks. The network is embedded in a hierarchical social structure in form of b-ary tree. One can imagine starting from the top level which represents the whole world, and the lower levels of the tree will correspond to countries, cities, etc down to the smallest social organization in which one people knows only one other. In such structure, the dis- tance between nodes is measured by the height of the nearest common ancestor. The authors proposed a method to generate networks from this structure by connecting randomly nodes at distance $x$ with probability $p(x)=c \exp -\alpha x$ where $\alpha$ is the parameter of the model and $c$ is a normalizing constant. Again, they found that there is only one value of $\alpha$ allowing a greedy search algorithm to perform in logarithmic time otherwise the delay is polynomial.

The above approaches work under the assumption that nodes know some information on the whole network or on the destination which are not necessarily true in the case of DTNs. In the context of DTNs, routing has drawn much attention (see [2] for a survey). Generally, we can classify the routing protocols in three categories: oblivious routing, mobility-based routing and social-based routing. In the first approach, nodes don't use any information on the network and either randomly forward the message or just flood it into the network. In the second approach, nodes leverage on the information on mobility like their position to make the forwarding decision (e.g, PROPHET [11]). The third approach consists in using the social information, e.g., node degree, detected communities, daily routines, to forward the message to the best candidate nodes (e.g., BubbleRap [5], dLife [13]). These informations are not always available or difficult to obtain.

In this work, we address a more general problem by not leveraging on the assumption on social structure or mobility. We postulate that mobile nodes may leverage on the intrinsic temporal structure that exists in every DTNs to efficiently route information. Intuitively, when looking for someone through a third person, people usually ask questions such as "when was the last time you met him/her?, Do you know someone who recently meets him/her ?" etc. Therefore, time is also a cue to consider for routing in DTNs. We address this issue in the next section.

\section{TEMPORAL STRUCTURE OF DTNS}

This section aims to introduce the temporal structure that exists inherently in DTNs and a model that captures the disorder degree of such networks. Dynamic networks such as DTNs, in contrast to static networks, evolve in temporal and spatial dimensions. Therefore, one of the most simple and natural structure that could emerge from a DTN could be given by a relation of temporal and spatial order. This consideration leads to study if it makes sense to consider the notion of spatio-temporal proximity between nodes in a DTN. Intuitively, we can consider that the more often a node is close to another node the smaller is its spatiotemporal distance from this node. Therefore this notion of spatiotemporal distance is defined from a statistical point of view according the time and space behavior of nodes. Moreover, this notion of distance can be recursively defined by considering the transitive closure of contacts between nodes. This consideration raises the following questions that will find answers in this paper:

- Is there any simple way to process in every node, just from its opportunistic contacts, its spatiotemporal distance to the other nodes?

- Can we use this notion and the resulting order relation entailed on the space of nodes to improve routing decisions in dynamic networks? 


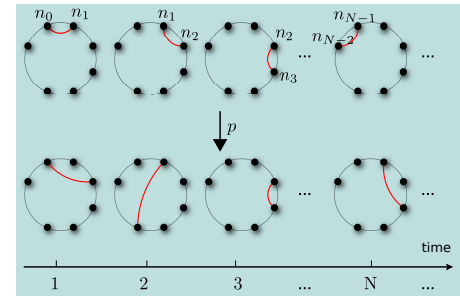

Figure 1: Network model

Following opportunistic contacts between nodes from a source to a destination along a dynamic path (as defined in [15]) every node can potentially process how far the source is from it. In other words every node of a dynamic path can be aware of the distance between the source and itself. Moreover, in [18], several traces analysis that we have driven lead to the conclusion that the spatio temporal distance in dynamic graph is symmetric. This means that during some time window the dynamic path length between node $i$ and node $j$ is equal to the dynamic path length between $j$ and $i$. Therefore as soon as nodes of a DTN can process their respective distance they can use this information during opportunistic contacts for assessing if a node in contact is "closer" or "farther" from a destination node. More formally, this notion of temporal distance is based on the two following metrics

- Delay $d_{i}^{j}(t)$ : the delay of node $i$ with respect to node $j$ at time $t$ is the elapsed time from the last moment when there was a dynamic path from $j$ to $i$. Formally,

$$
d_{i}^{j}(t)=\left\{\inf \left(t-t_{0}\right) \mid t_{0} \leq t \text { and } \exists\right. \text { a dynamic path from }
$$$$
\left.j \text { to } i \text { starting at time } t_{0}\right\} \text {. }
$$

- Hops $h_{i}^{j}(t)$ : the number of nodes on the path.

If mobile nodes in a DTN keep track of these metrics with respect to other nodes, the metric values will maintain an order relation between all the nodes in the network which represents a "temporal and spatial proximity" between nodes. In consequence, this order relation forms a gradient field from a node towards the others. It's worthy to note that this temporal structure is inherent to any dynamic network in which there are intermittent contacts between nodes.

To build such structure, we need to maintain two scalars $d_{i}^{k}(t)$ and $h_{i}^{k}(t)$ for each destination $k$ at each node $i$. Initially, the metrics are null because nodes do not know about each others. Through opportunistic contacts, nodes learn about the existing of other nodes and update their scalars. The algorithm to build and maintain the temporal structure is summarized in Algorithm 1. Indeed, when two nodes are in contact, the node which is farther from the destination in time (i.e., has a smaller value of $d$ ) learns the new shorter dynamic path and hence updates its delay value (i.e., equals to the other node's delay value) and its number of hops (i.e., equals to number of the other node plus 1 ). Note that the delay metric can be implemented by two methods. The straightforward implementation of the definition consists in using a timer to keep track of the delay with respect to a destination. We consider here a simpler method in which $d_{i}^{k}(t)=\left\{\sup \left(t_{0}\right) \mid t_{0} \leq t\right.$ and $\exists$ a path from $k$ to $i$ starting at time $\left.t_{0}\right\}$.

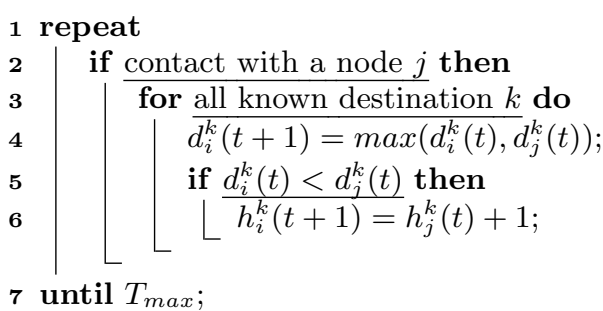

Algorithm 1: Building the temporal structure at node $i$

These two simple metrics reflect how a DTN is structured. Indeed, a DTN may be very regular, i.e., with a periodic contact pattern in which a node is always connected to the same node after the same time interval. In such network, the metric values are constant or periodic. On the contrary, the network regularity may be random such that a node can be connected to any node at any time. In this case, the metric values evolve randomly. We introduce in this paper a simple parametric model able to capture the regularity/disorder degree of DTNs. This model covers the full scope between a totally regular network and a totally random network by gradually increasing the disorder of network's contact patterns.

Let us consider a network of $N$ nodes $n_{0}, \ldots, n_{N-1}$ that evolves over time with a periodic contact pattern as follows. At time $0, n_{0}$ is connected to $n_{1}$; at time $1, n_{1}$ is connected to $n_{2}$; at time $2, n_{2}$ is connected to $n_{3}$ and so on. This contact pattern repeats until time $T_{\max }$ beyond which we no longer observe the network. Note that we consider that contact arrivals are atomic and so can be serialized.

We introduce disorder into this network by rewiring its links as follows. For each contact, with a probability $p$ we replace it by a contact between another pair of nodes. With probability $1-p$ we let the contact unchanged. We operate this rewiring process until time $T_{\max }$. We illustrate the process in Figure 1.

This rewiring process results in:

- When $p=0$, the network is totally regular and expresses a periodic contact pattern.

- When $p=1$, the network is totally random i.e. any contact can happen at any time.

- By varying $p$ between 0 and 1 , we gradually inject disorder into the network. Therefore the parameter $p$ quantifies the disorder degree.

\section{DISORDER DEGREE OF REAL HUMAN- CENTRICS DTNS}

In this section, we investigate various real traces of HumanCentric DTNs to estimate their disorder degree using the previously described model. To estimate the rewiring probability $p$ of real networks, we first need to identify the periodic contact patterns in the traces. We apply the following algorithm to detect the contact patterns and estimate the value of $p$. For each pair of nodes $(i, j)$, their contact times are collected and a time series $c_{i j}(t)$ in which $c_{i j}(t)=1$ if $i$ encounters $j$ at time $t$ and 0 otherwise is generated. The auto-correlation coefficients of these series

$$
\operatorname{Corr}_{i j}(k)=\sum_{i=1}^{T_{\max }-k} c_{i j}(i) c_{i j}(i+k)
$$




\begin{tabular}{llll}
\hline Dataset & Size (nodes/days) & Environment & $p_{\text {real }}(\%)$ \\
\hline Cambridge05 & $12 / 6$ & laboratory & 12.97 \\
Intel & $9 / 6$ & laboratory & 16.78 \\
Cambridge06 & $36 / 54$ & city & 22.17 \\
Infocom06 & $98 / 4$ & conference & 27.77 \\
MIT & $104 / 246$ & campus,city & 29.96 \\
Infocom05 & $41 / 4$ & conference & 34.96 \\
Milan & $49 / 19$ & university & 39.31 \\
\hline
\end{tabular}

Table 1: Estimated disorder degree of real dynamic networks

give us the number of contacts that are separated by $k$ time units. We then reorder the auto-correlation coefficients in descending order and select the second mode $M_{i j}$ for each pair of nodes (the first mode corresponds to $k=0$ ). The lag $k$ that gives this mode is the most frequent contact period. For each pair of nodes, we count the number of $k$-periodic contacts then sum up globally. Consequently, an estimate of $p$ is given by

$$
p_{\text {real }}=\frac{\text { contacts }-\sum_{i j} M_{i j}}{\text { contacts }} .
$$

We ran the algorithm on various traces $([19,12,10,3])$. These network traces contain recorded bluetooth connection activities in different environments. Table 1 shows the characteristics and the estimated $p_{\text {real }}$ values of all traces.

The results show that the analyzed Human-Centric DTNs exhibit a low disorder degree ranging from $10 \%$ to $40 \%$. We can see that the large traces seem to have a larger disorder degree than the small ones. Especially, spontaneous networks, such as networks at conferences, exhibit a higher randomness than business structured networks. The results also suggest that, the more a DTN is structured around a business activity, the more regular the network is.

\section{ROUTING IN HUMAN-CENTRIC DTNS USING TEMPORAL STRUCTURE}

\subsection{One-message Routing Algorithms Class}

Routing aims to find an efficient path from a node to another which minimizes some costs. That is, if a node $i$ wants to send a message $m$ to a node $j$, how do $i$ can find a path to $j$ which minimize some cost function. In this paper, we propose a simple and efficient solution using the temporal structure introduced in the previous section. As previously discussed, the temporal structure creates a order relation between nodes. Such order relation can be the cue allowing a node to find the efficient path. Indeed, when two nodes are in contact, they know which one is closer to the destination. Therefore the decision of forwarding the message to the destination can be based on this order relation.

A straightforward solution of routing in DTN is to send the message to every encountered node while keeping a message copy. This viral diffusion or flooding solution, while guaranteeing the best delay induces buffering and network capacity overheads, which make this solution impracticable in reality. In the following, for focusing on the impact of contact regularity on routing, we will consider the basic and worst case solution where only one copy of each packet is kept in the whole network.

Let us introduce a class of routing algorithms $\mathcal{A}$ that uses only one message copy reach the destination. Let assume

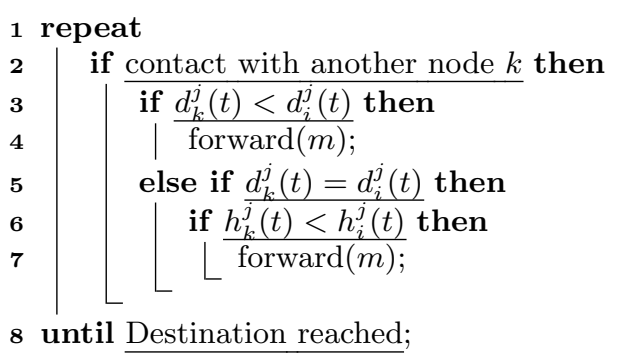

Algorithm 2: $G R A D-D O W N$

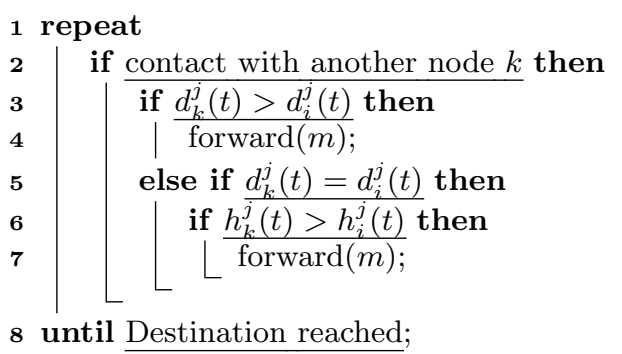

Algorithm 3: GRAD-UP

that at time $t$, a node $i$ search an efficient path towards a node $j$. It creates a message $m$ to $j$. At each contact with a node $k$, following the forwarding strategy of $\mathcal{A}, i$ will decide whether or not to forward $m$ to $k$. The goal of $\mathcal{A}$ is optimizing the cost for $m$ to reach $j$. In this paper, we consider 3 costs, respectively the delay, the delivery rate and the overhead which are 3 basic metrics to evaluate routing protocols in DTNs. We introduce here two greedy algorithms that exploit the temporal structure. In these algorithms, messages follow either upward or downward the gradient slope of the temporal structure to reach their destination. With the first algorithm named GRAD-DOWN, detailed in Algorithm 2, a node forward the message if the encountered node has a lower delay or an equal delay with a lower hops. Conversely, the GRAD-UP algorithm, detailed in Algorithm 3, consists in forwarding the message as soon as the encountered node has a higher delay or an equal delay with a higher hops. We will study the performance of these algorithms in function of the disorder degree of the network.

\subsection{Simulation Results \& Discussions}

In this section, we present and discuss our simulation results. We also provide a full formal analysis of the algorithm in [16]. To evaluate the performance of these algorithms, we implemented them in the ONE simulator for Delay Tolerant Networks [6]. We also compare them with 4 other algorithms: Direct Delivery, First Contact, PROPHET [11] and dLife [13]. Direct Delivery is the most radical solution in which a node waits until being in contact with the destination to deliver the message. FIRST-CONTACT consists in forwarding systematically the message at the first contact. The more elaborated PROPHET routing protocol is based on the contact history to infer the probability that a node will encounter the destination. Finally, dLife is a socialbased approach that exploits the human daily routines to deliver messages.

For a given rewiring probability $p$, we generate under MATLAB a regular connection trace of a network of 100 nodes with $T_{\max }$ being 10000 time units and rewire it fol- 


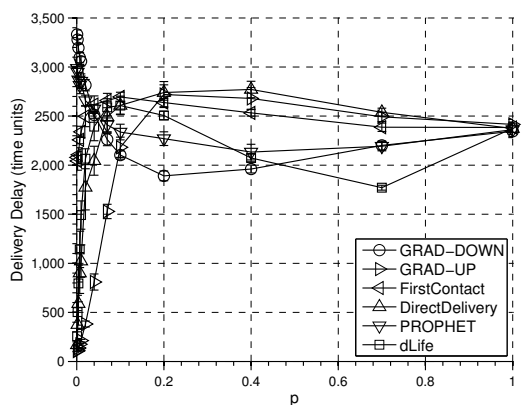

(a) Delivery delay

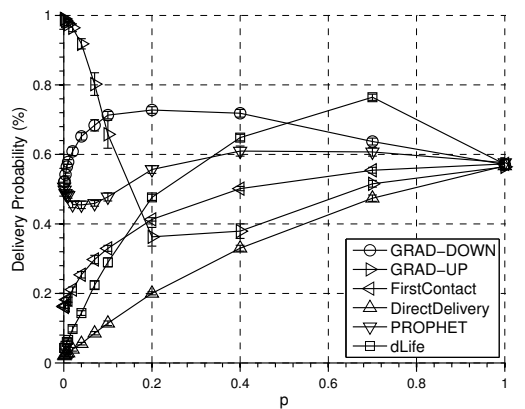

(b) Delivery rate

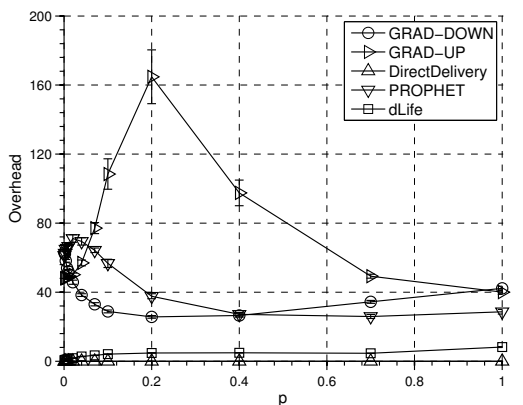

(c) Overhead

Figure 2: Performance comparison of all algorithms

lowing the model described in Section 3. The rewired trace is then fed to the ONE simulator [6]. As we want to focus only on measuring the impact of the disorder on these routing algorithms, the connection bandwidth and the buffers are assumed to be infinite. A message between a randomly taken pair of nodes is generated each 5 time units. Because our approach does not rely on an assumed daily routine, to be fair with dLife, we create a favorable simulation setting for this protocol by assigning a contact cycle to a day and a contact to a time slot.

We choose to evaluate the delivery delay, the delivery rate and the overhead (i.e., the number of relays to perform a successful delivery)-three basic performance measures-for all algorithms. For each value of $p$, we run each algorithm on 10 generated random traces. The performance is then measured as the average over 10 simulation runs. Figures $2(\mathrm{a}), 2(\mathrm{~b})$ and 2(c) depict the resulting three performance measures for all algorithms coupled with the $95 \%$ confidence intervals. We can see that the algorithms behaves differently as we vary the disorder degree in the network. With a highly regular network, GRAD-UP outperforms all other algorithms while Direct Delivery offers the worst performances among them. GRAD-DOWN and PROPHET perform quite bad in a regular network with a higher delivery rate and a lower overhead for GRAD-DOWN. FIRST-CONTACT and dLife deliver fast but with low delivery rate in a regular network. Moreover, the naive forwarding strategy of FIRSTCONTACT makes that its overheard is much higher than the others (in the order of $10^{4}$ ). For easy-reading, we do not plot this result.

When the disorder degree increases in the network, GRADDOWN outperforms all other algorithms by minimizing the delivery delay while maximizing the delivery rate and keeping a low overhead. As expected, the Direct Delivery algorithm performs worst in term of delivery delay and delivery rate due to its radical forwarding strategy. GRAD-UP and FIRST-CONTACT performs bad when the network is disordered. PROPHET also takes advantage of the disorder to deliver faster and with higher delivery rate than GRAD-UP and FIRST-CONTACT. dLife performs well in a highly disordered network, with slightly better performances in term of delivery delay and delivery rate compared to GRADDOWN. It's overhead is as good as Direct Delivery. This is a surprising result because dLife is supposed to work well in a regular network.

When the network is totally random, the algorithms have similar performances. In overall, GRAD-DOWN has the best performances over a wide range of network structures compared to the other algorithms. Its sibling GRAD-UP performs in an inverse manner. This first results show that the temporal structure creates a natural gradient field allowing, when exploited, the messages to reach their destinations with low delay, high delivery rate and low overhead. We will look closer at this phenomenon to understand how the temporal structure is formed and maintained in the network. In the following, we will focus on the delivery delay, which is an important performance measure for a routing algorithm in Human-Centric DTNs.

These results show that routing performances in DTNs greatly depends on the degree of disorder. Specifically when we introduce a little level of disorder into the network (i.e., $20 \%$ rewired links), the network become highly navigable using our approach. But the more disorder increases, the less we can leverage on time structure.

We can see that, in a totally regular network, the contacts follow the cycle: $n_{0} \leftrightarrow n_{1}, n_{1} \leftrightarrow n_{2}, \ldots, n_{N-1} \leftrightarrow n_{0}$. In consequence, according to the two previously introduced metrics and their updating procedures, when the contact between node $n_{i}$ and $n_{i+1}$ happens, $n_{i}$ is always "closer" to the destination $k$ than $n_{i+1}$ in term of delay and number of hops, and hence has a smaller gradient value. In that case, GRAD-UP achieves its best performance, as we can see on Figure 2(a) and 2(b), because the message is forwarded in the direction of the contacts. Conversely, GRAD-DOWN performs badly in this case because the node bearing the message has to wait a contact cycle to encounter a node whose the gradient value is smaller. By increasing the disorder degree in the network, we introduce shortcuts that shorten the contact cycle. GRAD-DOWN takes advantage of these shortcuts to make faster deliveries while GRAD-UP is penalized by them. At the other extrema, in a totally disordered network, any contact can happen at any time hence there is no longer interest in leveraging on order in the contacts. Too many shortcuts contribute to suppress the cue to find efficient paths resulting in message converging more slowly towards their destination. Intuitively, the optimal point (i.e. $p$ equals to 0.2 ) corresponds to a network structure in which the contact order is still conserved but the number of injected shortcuts is just enough to reduce the delay until being in contact with a node with smaller gradient value. We confirm this intuition by collecting the traces of messages which successfully arrived at their destinations. These traces contain the chains of nodes by which the message passed through and also the time when it was 


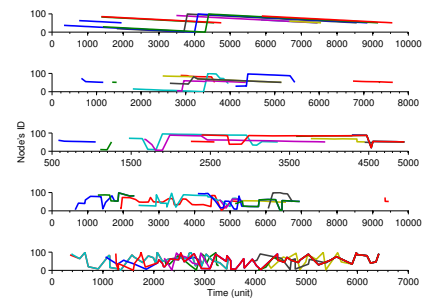

(a)

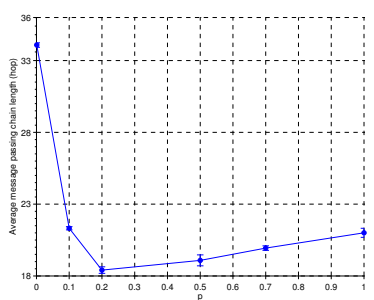

(b)
Figure 3: (a) Message passing chains (from the top, $p=0,0.1,0.2,0.5,1$ respectively) (b) Average message passing chain length (hops)

forwarded to these nodes. Figure 3(a) shows 10 chains for $p$ equals to $0,0.1,0.2,0.5,0.7$ and 1 . The $\mathrm{x}$-axis depicts the time and the y-axis depicts the node's IDs. A totally regular network makes the nodes holding a message to wait during 100 time units before forwarding it to another node. On the other hand, the algorithm does not perform better in a totally random network because the message goes back and forth between nodes. In consequence the delivery delay is high. A slight disorder improves significantly the performance thanks to the shortcuts that reduce the path length. To be more precise, Figure 3(b) shows the average chain length (i.e. number of nodes on the chain) over 10 simulation runs. It is clear that we achieve the best performances at $p$ equals to 0.2 .

\section{CONCLUSION}

This paper has analyzed the impact of the periodicity and regularity of nodes inter-contacts on information routing in Human-Centric DTNs. First, we gave the characterization of the spatio-temporal structure of these networks from the notions of spatio-temporal distance between nodes. Then, based on real dynamic network trace analysis, we showed that the inter-contact relations follow some degree of periodicity and regularity. We formalize these notions of regularity and periodicity of contacts via a simple parametric model. From these definitions, we studied the impact of the disorder, expressed as a decrease in the regularity and periodicity of contact patterns, on the performances of routing protocols and showed that a family of routing algorithms can leverage on this disorder to deliver messages efficiently. Especially, we showed that when a dynamic network has a fraction of disorder of about $20 \%$, this algorithm achieves its optimal performance. At the best of our knowledge, this is the first work that studies this aspect of DTNs. However, we are convinced that there are rooms to develop further this research. Especially, the model presented in this paper can be extended to be more realistic by considering different frequencies of contact for different nodes. One other possible direction is to combine the two presented routing algorithms to have an adaptive algorithm that performs efficiently in all contexts.

\section{REFERENCES}

[1] A. Clauset and N. Eagle. Persistence and periodicity in a dynamic proximity network. In DIMACS/DyDAn Workshop on Computational Methods for Dynamic Interaction Networks, 2007.
[2] M. Conti, J. Crowcroft, S. Giordano, P. Hui, H. Nguyen, and A. Passarella. Routing Issues in Opportunistic Networks. Middleware for Network Eccentric and Mobile Applications, 1:121, 2009.

[3] N. Eagle and A. (Sandy) Pentland. Reality mining: sensing complex social systems. Personal and

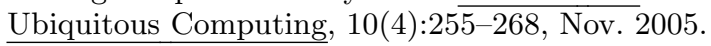

[4] P. Holme and J. Saramäki. Temporal networks. Physics Reports, 519(3):97-125, Oct. 2012.

[5] P. Hui, J. Crowcroft, and E. Yoneki. BUBBLE Rap: Social-Based Forwarding in Delay-Tolerant Networks. In ACM MOBIHOC, 2008.

[6] A. Keränen, J. Ott, and T. Kärkkäinen. The ONE simulator for DTN protocol evaluation. Simulation Tools and Techniques, pages 1-10, Mar. 2009.

[7] J. Kleinberg. Navigation in a small world. Nature, 406(6798):845, Aug. 2000

[8] J. Kleinberg. Complex networks and decentralized search algorithms. In Proceedings of the International Congress of Mathematicians (ICM), 2006.

[9] L. Kovanen, M. Karsai, K. Kaski, J. Kertész, and J. Saramäki. Temporal motifs in time-dependent networks. Journal of Statistical Mechanics: Theory and Experiment, Nov. 2011.

[10] J. Leguay, A. Lindgren, J. Scott, T. Friedman, J. Crowcroft, and P. Hui. \{CRAWDAD $\}$ data set upmc/content (v. 2006-11-17).

[11] A. Lindgren, A. Doria, and O. Schelén. Probabilistic routing in intermittently connected networks. ACM SIGMOBILE Mobile Computing and Communications Review, 7(3):19-20, 2003.

[12] P. Meroni, S. Gaito, E. Pagani, and G. P. Rossi. \{CRAWDAD\} data set unimi/pmtr (v. 2008-12-01).

[13] W. Moreira, P. Mesdes, and S. Sargento. Opportunistic routing based on daily routines. In IEEE WOWMOM, 2012.

[14] M. Newman. Networks: an introduction. Oxford University Press, 2010.

[15] A.-D. Nguyen, P. Senac, and M. Diaz. Understanding and modeling the small-world phenomenon in dynamic networks. In ACM MSWiM 2012, page 377, New York, New York, USA, Oct. 2012. ACM Press.

[16] A.-D. Nguyen, P. Sénac, and M. Diaz. How Disorder Improves Navigation in Dynamic Networks. Technical report, ISAE-University of Toulouse, Jan. 2013.

[17] A.-D. Nguyen, P. Senac, and M. Diaz. On the Impact of Disorder on Dynamic Network Navigation. In IEEE INFOCOM, Student Workshop, 2013.

[18] A.-D. Nguyen, P. Sénac, V. Ramiro, and M. Diaz. Swarm-based Intelligent Routing (SIR) - a New Approach for Efficient Routing in Content Centric Delay Tolerant Networks. In ACM MOBIWAC, 2011.

[19] J. Scott, R. Gass, J. Crowcroft, P. Hui, C. Diot, and A. Chaintreau. $\{$ CRAWDAD $\}$ data set cambridge/haggle (v. 2009-05-29).

[20] J. Travers and S. Milgram. An experimental study of the small world problem. Sociometry, 32:425-443, 1969.

[21] D. J. Watts, P. S. Dodds, and M. E. J. Newman. Identity and search in social networks. Science, 296(5571):1302-5, May 2002. 